\title{
Assessment of encapsulation materials for solar cells
}

\author{
Vladimír Šály, Vladimír Ďurman, Michal Váry, Milan Perný and František Janíček \\ Slovak University of Technology, Faculty of Electrical Engineering and Information Technology \\ Ilkovičova 3, SK-81219 Bratislava, Slovak Republic
}

\begin{abstract}
Interfacial processes were studied in various insulation foils intended for encapsulation of photovoltaic cells. The analysis was based on the dielectric measurements in a broad region of temperatures and frequencies. The measurements showed that the observed processes are connected with the electrode polarization. The electrode polarization gives rise to the space charge formation and enhancement of electric field near the electrodes. Calculation of the electric field is important for praxis as it allows assessing the risk of electrical breakdown. In our work we use the parameters obtained from the dielectric measurements for calculation of electric field distribution in encapsulating materials. It was found that electric field increases more than 100-times comparing with the mean value.
\end{abstract}

\section{Introduction}

Compact photovoltaic module is a layered structure consisting of interconnected and encapsulated solar cells embedded between substrate and superstrate (back and front sheets), glass or plastic sheets, in order to create compact and sealed unit protected against environmental influences. Solar cells strings are encapsulated between two sheets of encapsulant foil. Encapsulant is a polymeric material which also provides adhesion between substrate sheet and superstrate glass and strings of solar cells among them [1]. Photovoltaic module is source of electricity and from the electrical point of view encapsulant insulates electrically active solar cells and interconnecting wires on one side and also protects against direct electric contact in order to avoid electric hazard. Insulation properties and dielectric properties are among the important indicators tested at the beginning and also during lifetime of photovoltaic module [2]. Dielectric and electric tests are common to ensure the efficient operation and safety over time [3,4]. The encapsulant as an electric insulator has to withstand electric stress which when steadily increased, finally overcomes electric strength. The processes leading to electric breakdown are either quite rapid - short term or longer term caused by eg partial discharge activity or electrical treeing [5]. Electrical faults occurring in PV arrays as a result of electric breakdown are more hazardous that demand immediate attention [6]. In the case of many solar cells connected into series, the encapsulant is electrically stressed mainly at sharp edges of the structure. Another source of enhanced electric field is accumulation of space charge at the dielectric - electrode interfaces. This phenomenon is connected with the electrode polarization [7]. In the process 
of polarization the free cations in dielectric are accumulated near the negative electrode, creating a positive charge. A similar process runs near the positive electrode. The steady state of accumulation is given by the balance of concentration gradient and the Coulombic (electric) force $[8,9]$. The cations in dielectric and the negative charge at the electrode create so-called double layer. The layer is characterized by the Debye length:

$$
L_{D}=\frac{1}{q}\left(\frac{\varepsilon_{0} \varepsilon_{R} k T}{n_{0}}\right)^{1 / 2},
$$

where $q$ is the ionic charge, $\varepsilon_{R}$ is the relative permittivity of material in absence of free charges, $n_{0}$ is the free ion concentration, $k$ is Boltzmann's constant and $T$ is temperature. In the case of non-polar material the value of $\varepsilon_{R}$ equals to $\varepsilon_{\infty}$. (the optical permittivity). The whole process matches to the Debye relaxation function with the static permittivity of

$$
\varepsilon_{s}=\frac{L}{2 L_{D}} \varepsilon_{\infty}=\frac{L \varepsilon_{\infty}}{\frac{2}{q}\left(\frac{\varepsilon_{0} \varepsilon_{\infty} k T}{n_{0}}\right)^{1 / 2}} .
$$

For relaxation time $\tau_{0}$ the next equation is valid:

$$
\tau_{0}=\frac{L}{2 L_{D}} \frac{\varepsilon_{0} \varepsilon_{\infty}}{\sigma} .
$$

In the previous equations we used label $L$ for the electrode distance and $\sigma$ for the material conductivity.

\section{Encapsulants}

The matter of investigation were three different materials commonly used in PV module production as encapsulants of solar cells. They were namely ethylene vinyl acetate (EVA), polyvinyl butyral (PVB) and thermoplastic polyurethane (TPU). Their composition depends on the producer and the final product should be stabilized and resistant against environmental influences with appropriate electric properties. EVA is most frequently used. It is copolymer of ethylene and vinyl acetate. The properties of EVA are improved with additives. [10]. Some details of composition and the function of respective additives is discussed in [11]. PVB is mostly composed of $76 \%$ vinyl butyral, $22 \%$ vinyl alcohol and 2 $\%$ vinyl acetate [12] originally more intended for safety glass. TPU represents relatively new material exploited as photovoltaic encapsulant composed of softeners, solid segments and filling additives. The properties and advantages in comparison to the other encapsulants are more precisely discussed e.g. in [13].

\section{Experiment}

In our work we examined three types of encapsulating materials which are regularly used for solar cells. The dielectric measurements were carried out with emphasis on the region of very low frequencies, where the electrode polarization can be identified. The measured data were treated in the form of frequency dependence of the absolute value of impedance and its phase. The experiments were made by an impedance analyzer constructed at our department particularly for the range of very low frequencies. It is based on measuring the voltage on the divider consisting of the impedance normal and the measured object. The 
real and the imaginary part of the measured impedance are calculated by the Fourier transform of the mentioned voltages. This transform can also reveal a possible non-linear behavior of the measured structure. The actual frequency range of this equipment is from $0.001 \mathrm{~Hz}$ to $1 \mathrm{kHz}$. The voltage on the specimen during measurements was $5 \mathrm{~V}$. The specimens were investigated in the temperature range from about 30 to $80{ }^{\circ} \mathrm{C}$. During the measurements the temperature was stabilized in the thermostat TETTEX, type 2965. We used the stainless steel three-electrode system with electrode diameter of $5 \mathrm{~cm}$. The thicknesses of the foil specimens were: $0.50 \mathrm{~mm}$ (EVA), $0.36 \mathrm{~mm}$ (PVB) and $0.47 \mathrm{~mm}$ (TPU).

The measured values of impedance are compared by the means of the phase plots in Figs. $1-3$. The phase obviously changes from zero at low frequencies to $-\pi / 2$ at higher frequencies. In the case of electrode polarization, the phase approaches to $-\pi / 2$ on both sides of the frequency region. It gains a very flat maximum with the value near to zero. A dipolar polarization (if exists) causes a small maximum of phase in the medium or high frequency range. From the results of our measurements we can state, that no polarization can be identified in the EVA specimen. The phase in this material shows only a conduction behavior (Fig. 1). The electrode polarization can be seen in the PVB specimen with expressive maximum of phase at $0.1 \mathrm{~Hz}$ (Fig. 2). Electrode and dipolar polarizations are present in TPU foil where the small maximum of dipolar polarization is overlapped by the electrode process (Fig. 3).

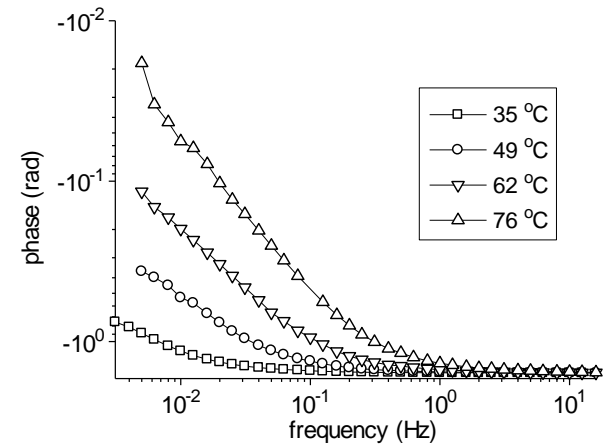

Fig. 1. Phase plot for EVA.

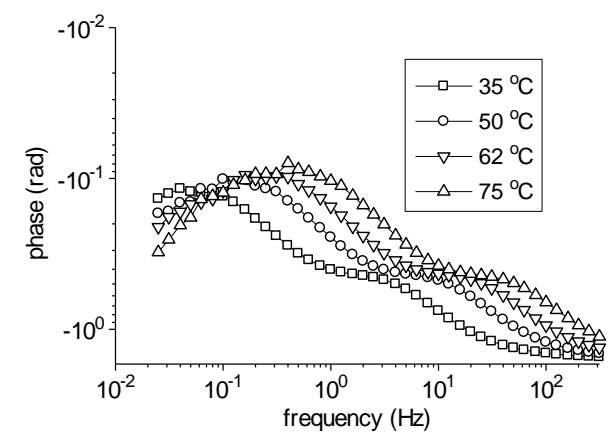

Fig. 3. Phase plot for TPU.

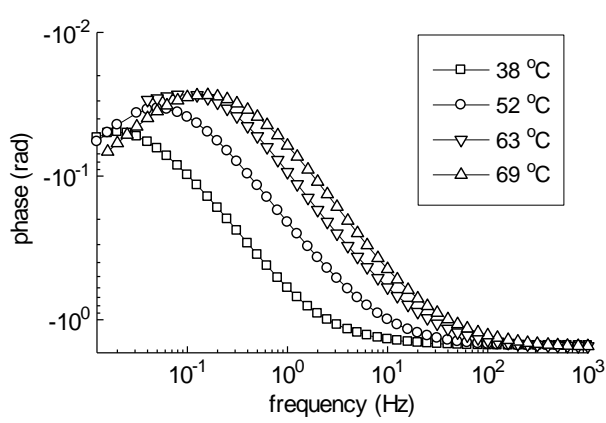

Fig. 2. Phase plot for PVB.

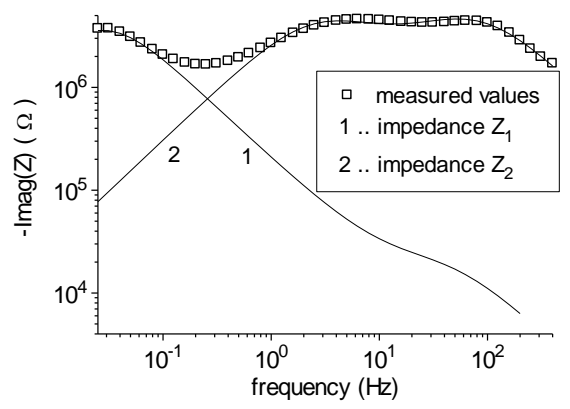

Fig. 4. Fitting functions - TPU $\left(62^{\circ} \mathrm{C}\right)$. 


\section{Results and discussion}

At modeling the dielectric processes in PVB and TPU encapsulating materials we come out from the assumption, that the thin Debye layer is arranged in parallel with the dielectric in the bulk of specimen. Both layers have the same dielectric parameters, but they differ in their conductivities. We are dealing with two impedances which are described with help of the well-known Cole-Cole formula [14]:

$$
Z_{I}=\frac{1}{\boldsymbol{j} \omega C_{0 I}\left(\varepsilon_{\infty}+\frac{\varepsilon_{s}-\varepsilon_{\infty}}{1+\left(\boldsymbol{j} \omega \tau_{0}\right)^{1-\alpha}}-\boldsymbol{j} \frac{\sigma_{I}}{\varepsilon_{0} \omega}\right)} .
$$

The index $I$ in the above formula acquires alternatively value 1 (for Debye layer) or 2 (for bulk layer). Here we used the next labeling: $\mathbf{j}$ is the imaginary unit, $\omega$ is the angular frequency, $\varepsilon_{\infty}$ is the optical permittivity of the dielectric, $\varepsilon_{0}$ is the vacuum permittivity, $C_{0}$ is the geometric or vacuum capacitance, $\varepsilon_{s}$ is the static permittivity, $\tau_{0}$ is the relaxation time, $\alpha$ is the shape parameter and $\sigma$ is the conductivity of dielectric. The unknown material parameters were calculated by fitting the measured values of the complex impedance $Z$ with the sum of impedances $Z_{1}$ and $Z_{2}$. This was done by means of the Nelder-Mead type simplex search method. An example of fitting the imaginary part of impedance for TPU foil at $62{ }^{\circ} \mathrm{C}$ is in Fig. 4. The complete calculated parameters are in Tab.1 (for PVB) and Tab. 2 (for TPU). The values are listed as functions of temperature $t$.

Table 1. Dielectric parameters of PVB encapsulating foil.

\begin{tabular}{|c|c|c|c|c|c|c|c|c|}
\hline $\begin{array}{c}\mathrm{t} \\
\left({ }^{\circ} \mathrm{C}\right)\end{array}$ & $\varepsilon_{\infty}$ & $\varepsilon_{\mathrm{s}}$ & $\begin{array}{c}\tau_{0} \\
(\mathrm{~s})\end{array}$ & $\begin{array}{c}\sigma_{1} \\
\left(10^{-12} \mathrm{Sm}^{-1}\right)\end{array}$ & $\begin{array}{c}\sigma_{2} \\
\left(10^{-9} \mathrm{Sm}^{-1}\right)\end{array}$ & $\begin{array}{c}\mathrm{h}_{1} \\
\left(10^{-7} \mathrm{~m}\right)\end{array}$ & $\begin{array}{c}\mathrm{h}_{2} \\
\left(10^{-4} \mathrm{~m}\right)\end{array}$ & $\mathrm{E}_{1} / \mathrm{E}_{2}$ \\
\hline 39 & 5.76 & 8.76 & 0.176 & 4.43 & 0.51 & 4.57 & 3.60 & 54 \\
\hline 45 & 5.76 & 8.67 & 0.118 & 6.14 & 0.89 & 1.81 & 3.60 & 144 \\
\hline 52 & 5.75 & 8.56 & 0.075 & 6.34 & 1.65 & 1.06 & 3.60 & 260 \\
\hline 58 & 5.75 & 8.47 & 0.052 & 7.46 & 2.75 & 0.78 & 3.60 & 369 \\
\hline 63 & 5.74 & 8.40 & 0.039 & 8.91 & 4.16 & 0.64 & 3.60 & 466 \\
\hline 69 & 5.74 & 8.32 & 0.027 & 1.13 & 6.70 & 0.52 & 3.60 & 590 \\
\hline 75 & 5.73 & 8.24 & 0.020 & 1.47 & 10.60 & 0.44 & 3.60 & 722 \\
\hline
\end{tabular}

Table 2. Dielectric parameters of TPU encapsulating foil.

\begin{tabular}{|c|c|c|c|c|c|c|c|c|c|}
\hline $\begin{array}{c}\mathrm{t} \\
\left({ }^{\circ} \mathrm{C}\right)\end{array}$ & $\varepsilon_{\infty}$ & $\varepsilon_{\mathrm{s}}$ & $\begin{array}{c}\tau_{0} \\
(\mathrm{~s})\end{array}$ & $\alpha$ & $\begin{array}{c}\sigma_{1} \\
\left(10^{-12} \mathrm{Sm}^{-1}\right)\end{array}$ & $\begin{array}{c}\sigma_{2} \\
\left(10^{-9} \mathrm{Sm}^{-1}\right)\end{array}$ & $\begin{array}{c}\mathrm{h}_{1} \\
\left(10^{-7} \mathrm{~m}\right)\end{array}$ & $\begin{array}{c}\mathrm{h}_{2} \\
\left(10^{-4} \mathrm{~m}\right)\end{array}$ & $\mathrm{E}_{1} / \mathrm{E}_{2}$ \\
\hline 35 & 6.61 & 37.8 & 0.135 & 0.07 & 2.17 & 1.74 & 18.0 & 4.69 & 80 \\
\hline 42 & 6.48 & 37.7 & 0.088 & 0.07 & 2.70 & 2.76 & 14.1 & 4.69 & 102 \\
\hline 50 & 6.34 & 37.6 & 0.055 & 0.08 & 3.59 & 4.56 & 11.2 & 4.69 & 127 \\
\hline 56 & 6.24 & 37.6 & 0.039 & 0.09 & 4.50 & 6.55 & 9.77 & 4.69 & 145 \\
\hline 62 & 6.14 & 37.5 & 0.028 & 0.09 & 5.67 & 9.27 & 8.64 & 4.69 & 163 \\
\hline 68 & 6.05 & 37.4 & 0.020 & 0.10 & 7.14 & 13.0 & 7.75 & 4.69 & 181 \\
\hline 75 & 5.95 & 37.3 & 0.014 & 0.11 & 9.33 & 18.9 & 6.91 & 4.69 & 202 \\
\hline
\end{tabular}

The value of $\alpha$ was negligible for PVB foil, so it is omitted in Tab. 1. Except the mentioned values, in the tables there are calculated thicknesses of Debye layer (assigned as $h_{1}$ ) and the bulk layer $\left(h_{2}\right)$. The last columns of the tables include the value of electric field $(E)$ enhancement in the Debye layer as $E_{1} / E_{2}$ which equals to $\sigma_{2} / \sigma_{1}$. This equality comes from the general principle of electric current continuity. 
The dielectric and electrode processes in the individual encapsulating materials can be assessed according to the value of conductivity and its activation energy. The conductivity obeys the well-known Arrhenius law, as it is seen in Fig. 5. The calculated activation energies are in Tab. 3.

The processes we have observed can be divided into the part of dipolar relaxation and the part of electrode polarization. The dipolar relaxation appears both in PVB and TPU foil. These processes were already known $[15,16]$. They are attributed to the retard motion of the polar side groups on the long polymer chain. When comparing the tested encapsulating materials according to their conductivities, apparently, the EVA foils provides the smaller conductivity with higher activation energy. Conductivity of EVA is approximately 1000 times lower than those of PVB and TPU. On the other hand, the relative high conductivities of the PVB and TPU foil results in the electrode polarization which enhances the local electric field. The thickness of the Debye layer is of the order $10^{-7} \mathrm{~m}$ while the conductivity at the electrode boundary is more than 100 times lower than in the bulk of material. As the boundary conductivity depends less on temperature than the bulk one, the difference against the bulk conductivity increases with temperature. The ratio of electric field in the Debye layer and the bulk layer in the steady state (dc voltage) is inversely proportional to the ratio of the layers conductivities. That is why the enhancement of electric field is more significant at higher temperatures. As it is seen from the last column of Tab.1 and Tab. 2, the enhancement has approximately linear character with slope 18 for PVB and 3 for TPU. The electrode process of course depends on the electrode material. The electric field enhancement in particular cases can differ from our observations which were realized on stainless steel electrodes.

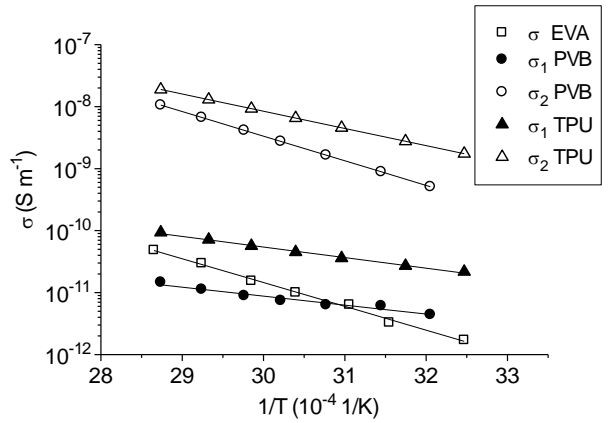

Table 3. Activation energies of conductivity.

\begin{tabular}{ccccc}
\hline \multicolumn{3}{c}{ Activation energy (eV) } \\
\hline \multirow{2}{*}{ EVA } & \multicolumn{2}{c}{ PVB } & \multicolumn{2}{c}{ TPU } \\
& $\sigma_{1}$ & $\sigma_{2}$ & $\sigma_{1}$ & $\sigma_{2}$ \\
\hline 0.76 & 0.29 & 0.79 & 0.34 & 0.55 \\
\hline
\end{tabular}

Fig. 5. Arrhenius plot of conductivity.

\section{Conclusion}

Three types of insulation materials intended for use as encapsulating foils of solar cells were studied. The investigations were based on the results of impedance spectroscopy. A special importance was attached to the region of very low frequencies. Except of the previously known dielectric relaxation, we detected appearance of electrode polarization in two materials, namely PVB and TPU. By analyzing the impedance data we found the thicknesses of the boundary layers and also their conductivities. These results are important at assessment of electric field distribution during the solar cell operation. The electric field in this configuration is inversely proportional to the ratio of the layers conductivities. At room temperature the field enhancement reaches the value of about 50. At higher temperatures, which are solar cells operational temperatures, increases the field approximately $200-600$ times. Although the insulating material is stressed only locally, deterioration due to enhanced stress may appear during a long-time operation. Inhomogenous electric field distribution represents increased probability to activate the 
processes leading to deterioration of insulator which can lead at critical level to electric breakdown process. That is why the material must be checked by accelerated aging tests performed at higher electric fields.

The acknowledgements: This work was supported by the Slovak Research and Development Agency (APVV) under the Contract No APVV-15-0326. This contribution is also the result of the project implementation: National Centre for Research and Application of Renewable Energy Sources (ITMS: 26240120016), supported by the Research \& Development Operational Programme funded by the ERDF.

\section{References}

1. R. Polanský, M. Pinkerová, M. Bartůn̆ková, P. Prosr, Mechanical behavior and thermal stability of EVA encapsulant material used in photovoltaic module, Journal of Electrical Engineering Vol. 64, 361 (2013)

2. M. De Oliveira, A. Diniz, M. Viana, V. De Freitas Cunha Linsa, The causes and effects of degradation of encapsulant ethylene vinyl acetate copolymer (EVA) in crystalline silicon photovoltaic modules: A review, Renewable and Sustainable Energy Reviews 81, 2299 (2018)

3. J. D. Lind, Photovoltaic panel impulse voltage testing, evaluation engineering, Jan. 2011 (https://www.evaluationengineering.com/photovoltaic-panel-impulse-voltagetesting)

4. G. Cibira, PV cells electrical parameters measurement, Journal of Electrical Engineering Vol. 6874 (2017)

5. B. Sultan, J. Andersonn, T. Hjertbeg, Electrical breakdown testing of materials intended for use in PV modules, (3rd Atlas/NIST Workshop on Photovoltaics Materials Durability. Gaitherburg, Maryland December 8-9, 2015)

6. D. S. Pillai, N. Rajasekar, A comprehensive review on protection challenges and fault diagnosis in PV systems. Renewable and Sustainable Energy Reviews 91, 18 (2018)

7. R. Coelho: Sur la relaxation d'une charge d'espace. Revue de Physique Appliquée 18, 137 (1983)

8. S. Emmert, M. Wolf, R. Gulich, S. Krohns, S. Kastner, P. Lunkenheimer, A. Loidl, Electrode polarization effects in broadband dielectric spectroscopy. European Physical Journal B83, 157 (2011)

9. J.R. Klein, S. Zhang, S. Dou, B.H. Jones, R.H. Colby, J. Runt, Modeling electrode polarization in dielectric spectroscopy. Journal Chemical Physics 124, 144903 (2006)

10. K. Agroui, G. Collins, Characterisation of EVA encapsulant material by thermally stimulated current technique, Solar Energy Materials and Solar Cells 80, 34 (2003)

11. De Oliveira, M.C.C. et al., The causes and effects of degradation of encapsulant ethylene vinyl acetate copolymer (EVA) in crystalline silicon photovoltaic modules: A review. Renewable and Sustainable Energy Reviews 81, 2299 (2018)

12. A. K. Dhaliwal, J. N. Hay, The characterization of polyvinyl butyral by thermal analysis, Thermochimica Acta 391, 245 (2002)

13. G. Stollwerck, H. G. Stevens, H. J. Goerkes, W. Kaufhold, A. Lyding, H. Peerlings, E. U. Reisner, J. Ufermann, M. Vogt, Vacuum-free lamination of solar modules, (Proc. 20th European Photovoltaic Solar Energy Conference, 2002, 2005) 
14. M. Wubbenhorst, J. Vanturnhout, Analysis of Complex Dielectric Spectra. I. Onedimensional derivative techniques and three-dimensional modelling, Journal of NonCrystalline Solids 305, 40 (2002)

15. A.M. North, J.C. Reid, Dielectric relaxation in a series of heterophase polyether polyurethanes. European Polymer Journal 8, 1129 (1972)

16. B.L. Funt, Dielectric dispersion in solid polyvinyl butyral. Canadian Journal of Chemistry 30, 84 (1952) 